\title{
Minimal Resistance of Engineering Materials to Brittle Fracture as Predicted by Local Approach
}

\author{
A.J. Krasowsky and G. Pluvinage* \\ Institute for Problems of Strength, National Academy of Science of Ukraine, 2 Timiryazevskaya str., \\ Kiev 252014, Ukraine \\ * University of Metz, Ile du Saulcy, 57045 Metz cedex, France
}

\begin{abstract}
Two basic concepts of brittle fracture are considered: the first involving stage-by-stage interatomic bonds decohesion at the crack tip, the second - microcrack coalescence. Quantitative evaluation has been made of the material threshold and minimal fracture toughness values which correspond to the above concepts. The paper presents the analysis of methods for the experimental evaluation of two main structure parameters, which govern the material fracture, and discloses their physical meaning. These are the characteristic distance and cleavage stress. It also considers the methods of a priori express-evaluation of the materials fracture toughness from their conventional mechanical characteristics.
\end{abstract}

\section{INTRODUCTION}

According to the classical concept the material fracture process involves a stage-by-stage decohesion of interatomic bonds at the crack tip (decohesion model). The elemental crack extension occurs due to the act of decohesion of the interatomic bond nearest to the crack tip. The elemental distance to which it extends in one step is prescribed by the material atomic structure and the process continues step-bystep until the body separates into parts. The known classical brittle fracture theories, beginning with the theories of Griffith [1], Barenblatt [2], Leonov and Panasyuk [3] and others, consider (explicitly or implicitly) the fracture process to develop in that way. As a result, in the basic relationships of those theories the interpretation of the material fracture toughness is related to the atomic or molecular structure of a substance (surface energy, strength of interatomic bonds, forces of intermolecular cohesion, etc.).

Another conception of brittle fracture consider a sequence of acts of microcrack nucleation near the tip of the main crack and their subsequent coalescence. In contrast to the conception of "decohesion" model, we shall call it the concept of "coalescence" model, having in mind that without specifying the atomic mechanism of microcrack nucleation this conception implies that the conditions at the crack tip and the material structure ensure the initiation and propagation of a microcrack in the direction opposite to that of the main crack to their complete coalescence, Fig. 1. It should be noted that while decohesion fracture is seldom encountered in "a pure form" (e.g. cleavage of crystals), the coalescence fracture is characteristic of most engineering and other materials. This fact does not diminish the significance of "decohesion" fracture which is an important element of the "coalescence" mechanism at the stage of microcrack propagation. However, the fact that the material structure dictates precisely the "coalescence" mechanism which, as it will be shown here, requires higher fracture energy than the "decohesion" mechanism, predetermines its great practical importance since fracture toughness holds a special place among mechanical properties of engineering materials owing to its unique capability to characterize the capacity of the matter to resist crack propagation.

\section{THE "COALESCENCE" FRACTURE MODELS}

According to the local approach conception the condition of the brittle or ductile separation of material in the vicinity of crack tip can be presented in the form of some functional [4] 


$$
\mathrm{F}\left(\sigma_{\mathrm{ij}}, \varepsilon_{\mathrm{ij}}, \chi\right)=0 \ldots \text { at } \ldots \mathrm{r}=\mathrm{X}_{\mathrm{c}}
$$

where $\sigma_{\mathrm{ij}}, \varepsilon_{\mathrm{ij}}$ are the components of stress and strain tensors, $\chi$ is an appropriate set of the structural parameters and mechanical charactristics of material, $\mathbf{r}$ is the distance from crack tip and $X_{c}$ is some characteristic distance related to the material structure. Orowan [5] was the first to formulate a hypothesis on the possibility of fracture initiation at a certain distance ahead of the notch tip. It was

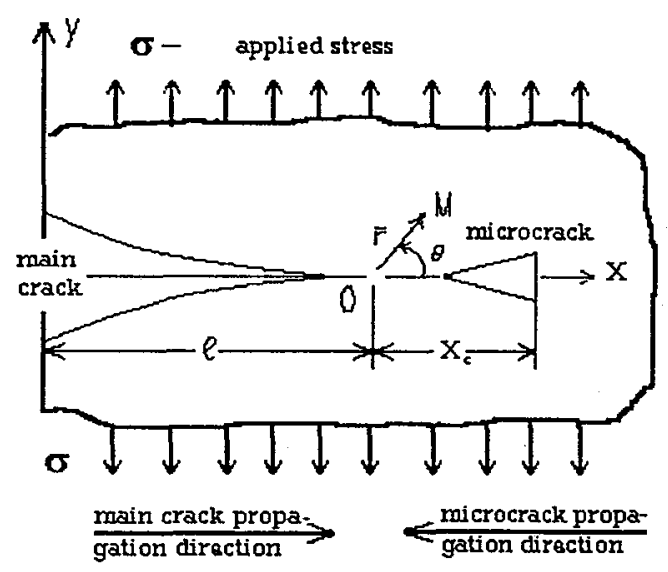

Figure 1: A schematical interpretation of the "coalescence" brittle fracture mechanism. Elemental crack advance is equal to characteristic distance $X_{c} \gg a$ ( $a$ is an interatomic distance).

based on the principal tensile stress distribution, calculated using the slip line theory, which exhibited a peak not at the very tip of the notch but ahead of it. Since the location of the stress peak depends on both the notch geometry and the magnitude of the acting load and is not predetermined solely by the material structure, the distance from the notch tip to the site of fracture initiation cannot be considered as the materials' structure parameter. Analogous remark can be made about a similar fracture criterion proposed by Neuber [6] and later by Novozhilov V.V., where the distance ahead of the notch tip, at which the stresses are averaged, is often called a structure parameter. Tetelman et al. [7] proposed a model for the notched bar brittle fracture with the stress distribution at the notch tip based on the slip line field analysis.

The model of "coalescence" brittle fracture involving the propagation of a sharp crack in hardening material was originally proposed in [8] (see also [9]). Based on the known [10-12] elasto-plastic solution of the mode I crack problem, the model incorporates two parameters of the material structure: characteristic distance $X_{c}$ from the crack tip to the site of microcrack nucleation and microcleavage stress $\sigma_{f}^{*}$, the local stress at a distance $X_{c}$ wich initiates fracture. Combining this parameters and the small scale yielding stress distribution [10-12] gives the dimensionless form of the main relationship of the model [8]

$$
\frac{\mathrm{K}_{\mathrm{I}}}{\mathrm{K}_{\mu}}=\frac{\sigma}{\sigma_{\mathrm{f}}^{*}}\left(\frac{\sigma}{\sigma_{y}}\right)^{\frac{1-\mathrm{n}}{2 \mathrm{n}}}
$$

which relates the stress intensity factor $K_{1}$, depending on the external load and the body geometry, with the local stress $\sigma$ acting at a distance $X_{c}$, yield stress $\sigma_{y}$ and the strain hardening exponent $n$. For convenience it is assumed that $\mathrm{K}_{\mu}=\sigma_{\mathrm{f}}^{*} \sqrt{\pi \mathrm{X}_{\mathrm{c}}}$ and the model is called the $K_{\mu}$-model. At the instant of fracture initiation the global $\left(K_{\mathrm{I}}=K_{\mathrm{Ic}}\right)$ and local $\left(\sigma=\sigma_{f}^{*}\right)$ fracture criteria should be satisfied simultaneously, so ultimately the model predicts the relation

$$
\frac{\mathrm{K}_{\mathrm{Ic}}}{\mathrm{K}_{\mu}}=\left(\frac{\sigma_{\mathrm{f}}^{*}}{\sigma_{\mathrm{y}}}\right)^{\frac{1-\mathrm{n}}{2 \mathrm{n}}}
$$


When deducing those relationships there was no need to make any assumptions concerning the nature of the $X_{c}$ and $\sigma_{f}^{*}$ values. However, in the calculation of fracture toughness, $K_{\mathrm{I} c}$, dependence on the temperature, $T$, and loading rate, $\dot{\mathrm{K}}$, within the framework of the proposed model, in [8] it was assumed unambiguously that $X_{c}$ and $\sigma_{f}^{*}$ are independent of the temperature, $T$, and strain rate, $\dot{\varepsilon}$, i.e. from the very beginning in the $K_{\mu}$ - model parameters $X_{c}$ and $\sigma_{f}^{*}$ were related to the material structure. The calculation revealed a natural character of the predicted $K_{\mathrm{I} c}(\mathrm{~T}, \dot{\mathrm{K}})$ dependence.

Two years later Ritchie, Knott and Rice [13] proposed a similar model in which they tried to take into account geometrical changes of the crack tip but did not obtain the analytical relation between $K_{\mathrm{I} c}$ and the values $X_{c}$ and $\sigma_{f}^{*}$ in the explicit form. The model proposed by Curry in 1976 [14] is based on the same prerequisites as the $K_{\mu}$-model and yields relationship (3) up to a constant factor, if in the Curry model the strain hardening exponent by Ramberg-Osgood is substituted by that adopted in the $K_{\mu}$-model, i.e. $N=1 / n$. The important probabilistic formulations of the local approach to brittle fracture have been done by Beremin [15] and Wallin [16] .
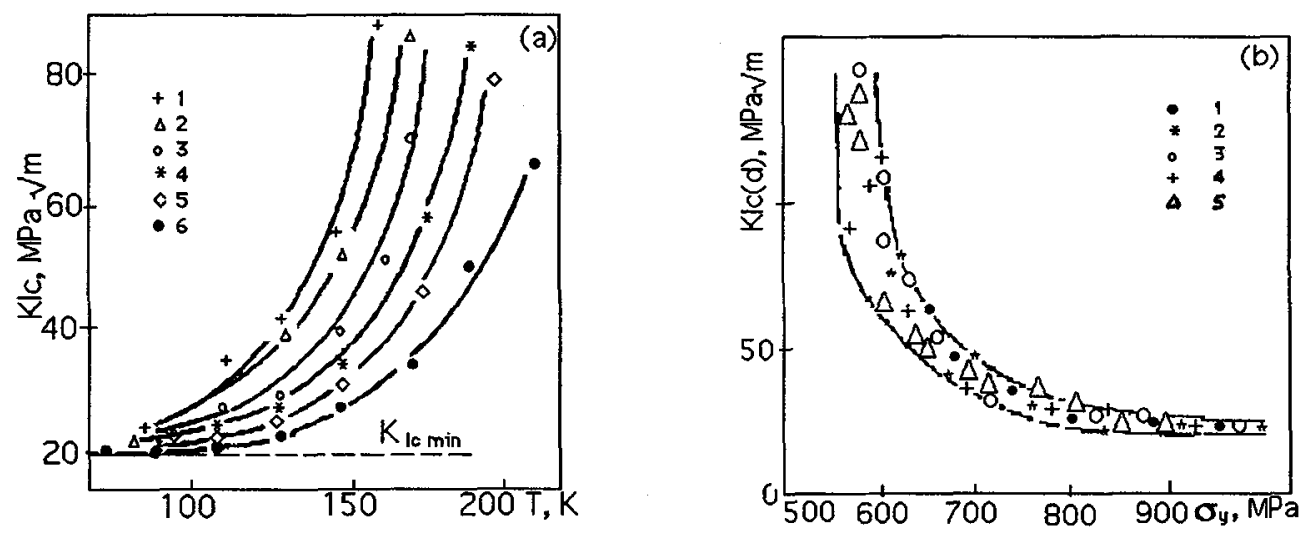

Figure 2: Fracture toughness of pipe-line steel $10 \mathrm{G} 2 \mathrm{PhB}$ (similar to $\mathrm{X} 70$ ) at different temperature-rate conditions.

a) Temperature dependence of $K_{\mathrm{I} c}$ at different loading rates, $\dot{\mathrm{K}}, \mathrm{MPa} \sqrt{\mathrm{m}} \cdot \mathrm{s}^{-1}: 1-4.3 \cdot 10^{\circ} ; 2-4.3 \cdot 10^{1} ; 3-4.2 \cdot 10^{2}$;

4- $3.7 \cdot 10^{3} ; 5-1.9 \cdot 10^{4} ; 6-(1 . .3) \cdot 10^{5}$. b) Relation between $K_{\mathrm{I} c}$ and yield point at different strain rates, $\dot{\varepsilon}, s^{-1}: 1-$ $8 \cdot 10^{-4} ; 2-8 \cdot 10^{-3} ; 3-8 \cdot 10^{-2} ; 4-8 \cdot 10^{-1} ; 5-8.10^{0}$.

First and foremost, basing on relation (3), one can make some useful conclusions. In particular, considering the assumed independence of the $X_{c}$ and $\sigma_{f}^{*}$ parameters of the temperature and strain rate, one can predict the relationship between $K_{\mathrm{I} c}$ and $\sigma_{y}$ in the form

$$
\mathrm{K}_{\mathrm{Ic}}=\mathrm{A} \sigma_{\mathrm{y}}^{\mathbf{n}^{\prime}}
$$

were $A$ is the factor comprising parameters $X_{c}$ and $\sigma_{f}^{*}$, and $n^{\prime}=-\frac{1-n}{2 n}$. This relation was indeed experimentally observed many times (see e.g. [9] or [17] and Fig. 2b) though only in those cases when $\sigma_{y}$ variation was not connected with the variation of fracture micromechanism parameters (e.g. in fracture toughness testing of steels in the brittle fracture region at different temperatures and loading rates, Fig.2, parameters $X_{c}$ and $\sigma_{f}^{*}$ remain unchanged). In contrast, when $\sigma_{y}$ variation is associated with structural changes (e.g. heat treatment), this causes changes in $X_{c}$ and $\sigma_{f}^{*}$ parameters, therefore no correlation is observed between $K_{\mathrm{I} c}$ and $\sigma_{y}$. 
Then relationship (3) predicts the existence of the so-called "lower shelf" or lowtemperature plateau on the material fracture toughness temperature dependence. Indeed, with a decrease in temperature (or with an increase in the loading rate) the size of the plastic zone at which fracture occurs is ever decreasing. Finally, the temperature can be lowered to such level that the size of the critical (i.e. at the instant of fracture) plastic zone becomes equal to the $X_{c}$ value. In this case the site of the microcrack nucleation is on the elasto-plastic boundary, hence $\sigma_{y}=\sigma_{f}^{*}$. For this reason, it follows from $\mathrm{Eq}(3)$ :

$$
\mathrm{K}_{\mathrm{Ic} \min }=\mathrm{K}_{\mu}
$$

i.e. $K_{\mu}$ in expression (3) is the lower limit of the fracture toughness of the material whose structure triggers the fracture micromechanism which is characterized by parameters $X_{c}$ and $\sigma_{f}^{*}$. It can be shown that with a further reduction of the critical plastic zone size and the fracture micromechanism remaining unchanged condition (5) will be still valid, i.e. relationship (3) predicts the existence of a low-temperature plateau on the fracture toughness temperature dependence which is generally observed in experiments [17].

\section{Physical meaning of PaRAmeters $x_{c}$ AND $\sigma_{f}^{*}$}

The data of fractographic investigations provide the experimental evidence of the existence of the "coalescence" fracture mechanism and hence, of the characteristic distance $X_{c}$. With low-carbon steels, for instance, fracture initiates, generally, at the grain boundaries and, as can be judged from the cleavage pattern orientation, the direction of its local extension is independent of the direction of the main crack propagation $[9,18]$, i.e. within each grain there is almost equal probability for fracture to develop both in the direction of the main crack propagation and opposite it.

As to the physical meaning of the characteristic distance, $X_{c}$, it is related to the structure of the material studied and can correlate with linear dimensions of one or another structure element. In the specific case of the low-carbon steel fracture by cleavage at low temperatures, the above features evidence that such structure element for the materials in question can be a grain with whose size the $X_{c}$ value may correlate even though a microcrack is nucleated on the grain boundary carbide. However, one should consider that relation (3) on which the $X_{c}$ estimation is based was obtained on a rather simplified assumption of the $K_{\mu}$-model, namely: a microcrack is nucleated at the $X_{c}$ distance ahead of the crack tip. In reality, intricate morphology of the steel brittle fracture surfaces indicates that cleavage facets are distributed in size approximately in accordance with the grain size distribution $[19,20]$; the facets orientations are also distributed in space in a certain manner. Thus, in addition to the $K_{\mu}$-model assumptions the $X_{c}$ parameter is of statistical nature and, strictly speaking, one should take into account the distribution of the $X_{c}$ local values both in size and in space orientations. However, these fractographic experimental facts provide the definite conclusion: at least for pure polycrystal materials (e.g. low-carbon steel) the characteristic distance cannot physically be larger than the grain size $[9,18]$. This conclusion contradicts some work (e.g. [13]) which considered the characteristic distance, $X_{C}$, as a model fitting parameter, and evaluated $X_{c}>1 d$, where $d$ is a grain size.

Physical meaning of the microcleavage stress $\sigma_{f}^{*}$ was discussed in detail in ref. $[21,22]$ and in many other works. For this reason we shall touch briefly on this problem only within the limits of its interpretation by the $K_{\mu}$ - model. The $\sigma_{f}^{*}$-value has a simple enough definition in the framework of the model: this is a local principal tensile stress $\sigma_{y y}$ ahead of the crack tip which is sufficient to induce a microcrack nucleation and propagation up to the length $X_{c}$ in the direction opposite to that of the main crack, Fig.1. However, experimental determination of this characteristic is not simple due to the fact that in its nature $\sigma_{f}^{*}$ is a local stress [23] and has a statistical nature [24]. Its determination is related to the determination of another parameter of the model, $X_{c}[23]$.

One of the methods of $\sigma_{f}^{*}$ - value determination follows from the above discussion of relationship (5): $\sigma_{f}^{*}$ is equal to the yield stress at the temperature at which fracture occurs with the critical plastic zone size being equal to $X_{c}$. However, it should be noted that $\sigma_{y}$ implies physical yield stress, i.e. that which corresponds to the stress state at the distance $X_{c}$ from the crack tip. The calculations [22] show that depending on the notch radius the stress peak ahead of the notch tip at a corresponding 
temperature can exceed the offset yield stress $\sigma_{0.2}$ for the uniaxial stress state $2 \ldots 2.5$ times. For the crack this excess may reach 3 times and more.

\section{EXPERIMENTAL EVALUATION OF PARAMETERS $x_{c}$ AND $\sigma_{f}^{*}$}

Relationship (5) gives the first method for estimating the $K_{\mu}$ value, which relates parameters $X_{c}$ and $\sigma_{f}^{*}$ from the experimentally obtained fracture toughness temperature dependence as the $K_{\text {Ic min }}$ values on the low-temperature shelf (see Fig. 2a). This method can be used to verify the correctness of the parameters $X_{c}$ and $\sigma_{f}^{*}$ estimated by other methods, since in the low-temperature range the following equality should be observed

$$
K_{\text {Ic } \min }=K_{\mu}=\sigma_{f}^{*} \sqrt{\pi \mathrm{X}_{\mathrm{c}}}
$$

or to obtain the magnitude of any one parameter, with the other parameter and the $K_{\text {Ic min }}$ values being known.

One of the first experimental evaluations of parameters $X_{c}$ and $\sigma_{f}^{*}$ for low carbon steel has been done in 1973-74 in Tohoku University. The results of this investigation have been published in work [25]. The comprehensive thermodynamic definition of the temperature and strain rate influence upon the plastic flow stress as well as upon the fracture toughness, $K_{\mathrm{Ic}}$, of low carbon steel with the grain size $d=0.034$ $\mathrm{mm}$ revealed the relation (4) with $\mathrm{n}^{\prime}=-1.5$, which corresponds to the work hardening exponent $n=$ 0.25. It follows from this work [25] that in the low temperature plateau range the value of $K_{\text {Ic min }} \leq 20$ $\mathrm{MPa} \sqrt{\mathrm{m}}$. Taking into account the value $X_{c} \leq d=0.034 \mathrm{~mm}$ one can get from Eq. (5)

$$
\sigma_{f}^{*}=\frac{\mathrm{K}_{\mathrm{Icmin}}}{\sqrt{\pi \mathrm{X}_{\mathrm{c}}}} \cong \frac{20}{\sqrt{\pi 34 \cdot 10^{-6}}} \cong 2000 \mathrm{MPa}
$$

which is the reasonable value of cleavage stress for low-carbon steel.

The $K_{\text {Ic min }}$ estimation can be performed by an independent though a more complex method involving precise stereoscopic measurements of the stretched zone height by superimposing of the mating fracture surfaces and comparison received height with the crack opening displacement, Fig.3. This method giving low scattering of results was proposed elsewhere [17]. It should be noted that verification of relation (6) against the data obtained from the reliable evaluation of $X_{c}$ and $\sigma_{f}^{*}$ by other methods yields fairly reasonable results. Thus, ref. [22] dedicated to the results of the interlaboratory experimental determination of $X_{c}$ and $\sigma_{f}^{*}$ presents fracture toughness temperature dependences for the 20MnMoNi55 and FeS10-CaSi steels from which $K_{\text {Ic min }}$ can be obtained equal to 25 and $25 \ldots 30 \mathrm{MPa} / \mathrm{m}$, respectively. The values $\sigma_{f}^{*}=2155 \mathrm{MPa}, X_{c}=30 \mathrm{~mm}$ (plate $\mathrm{N} 24$ ) and $\sigma_{f}^{*}=2032 \mathrm{MPa}, X_{c}=40$ $\mathrm{mm}$ (plate N25) for the former steel and $\sigma_{f}^{*}=1650 \mathrm{MPa}, X_{c}=100 \mathrm{~mm}$ for the latter one found in ref.[22] and used for $K_{\mu}$ estimation by formula (6) yield the estimates equal to 21 and $23 \mathrm{MPa} / \mathrm{m}$ for the former and $29 \mathrm{MPa} / \mathrm{m}$ for the latter steels which agree fairly well with extrapolated $K_{\text {Ic min }}$ values. Similar agreement was reported by Kotilainen [21] in his thorough investigations of the Cr-Mo-V steel with a bainite structure. Making use of the above data, let us elucidate the $X_{c}$ estimate obtained in ref.[17] using relationship (6). In this work $\sigma_{\mathrm{f}}^{*}$ was taken as $\sigma_{\mathrm{fc} \max }=1240 \mathrm{MPa}$ which corresponded to the yield stress extrapolated to the absolute zero temperature as it had been proposed by Aurich [22]. However, as indicated by numerous results $[21,22]$ for sharp cracks this method yields underestimated $\sigma_{f}^{*}$ values. For instance, for the three states steel studied in ref.[21] the average magnitude of the $\sigma_{f}^{*} / \sigma_{f c \max }$ ratio proved equal to 1.71 . If we correct the $1240 \mathrm{MPa}$ value by this multiplier, we get $\sigma_{f}^{*}=2125 \mathrm{MPa}$. Using the latter and formula (6) we obtain a new estimate $X_{c}=28 \mu \mathrm{m}$ instead of $82 \mu \mathrm{m}$ obtained earlier, which agrees better with the ferrite grain size distribution from ref. [17]. It should be also noted that the value $\sigma_{f}^{*}=2125 \mathrm{MPa}$ agrees better with other $\sigma_{f}^{*}$ estimates for other steels of similar strength. 
We can also give other experimental evidence which confirm the validity of relation (6) for sharp cracks. Within the framework of the $K_{\mu}$ - model this result seems to be logical since the $K_{\text {Ic min }}$ values for the low-temperature region correspond to fractures with very small plastic zones (or without them), when the crack tip plastic blunting which is not taken into account by the $K_{\mu}$-model is minimal.

Let us return to the problem of two brittle fracture conceptions and try to consider it from the quantitative standpoint. As it has already been noted, the $K_{\mathbf{I c} \text { min }}$ estimation based on the obtained $X_{c}$ and $\sigma_{f}^{*}$ parameters leads to a correlation with the $K_{\mathbf{I c} \text { min }}$ values corresponding to the lowtemperature shelf of the $K_{\mathrm{Ic}}(T)$ dependence. It is pertinent to ask what minimal fracture toughness values can be expected in the case of "decohesion" fracture micromechanism. We shall try to get the

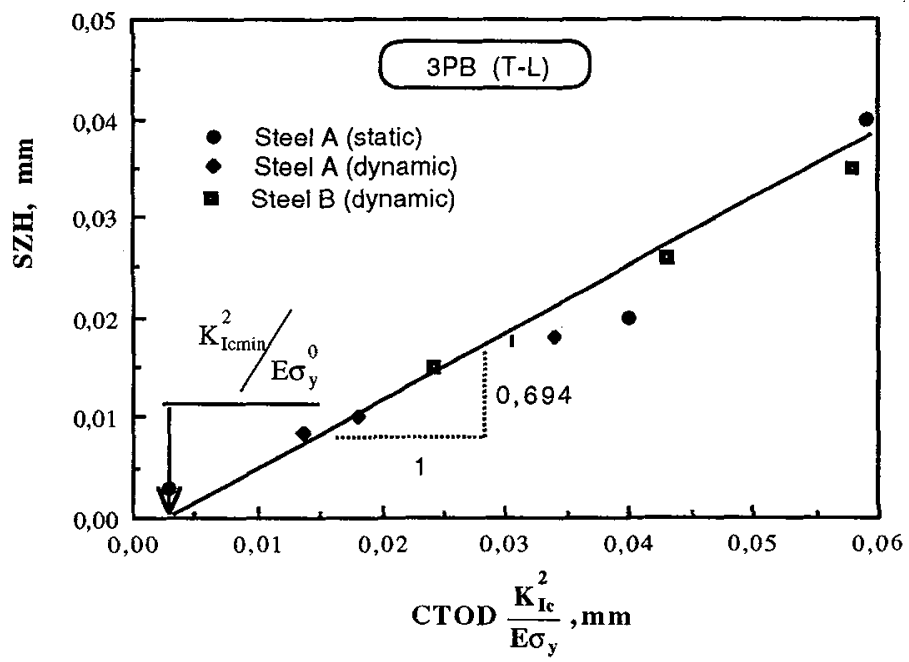

Figure 3: Relation between the measured values of the stretched zone height, SZH, and crack tip opening displacement, CTOD, estimated via $K_{1 C}$ within the temperature range of brittle and quasi-brittle fracture. Pipe-line steels of X70-type [17].

answer within the framework of the same $K_{\mu}$ - model. Let us assume that the model allows the "decohesion" fracture micromechanism to be considered as a particular case of the "coalescence" fracture micromechanism, namely, when the characteristic distance $X_{c}$ is equal to the interatomic distance $a$. In this case, the theoretical strength generally measured in fractions $\alpha$ of the elasticity modulus can be taken as the microcleavage stress. Using formula (6), calculate the fracture toughness threshold value $K_{\text {Ic } t h}$ which corresponds to the fracture mechanism mentioned:

$$
K_{\mu}=K_{\text {Ic } t h}=\alpha E \cdot \sqrt{\pi \mathrm{a}}
$$

Considering that generally $\alpha \cong 0.1$ and for steels $a \cong 2.5 \ldots 3 \dot{\mathrm{A}}$ we get $\left(K_{\mathrm{Ic}} / E\right)_{t h}=3 \cdot 10^{-6} \sqrt{\mathrm{m}}$, or adopting the elasticity modulus value common with steels, we get $K_{\text {Ic } t h}<1 \mathrm{MPa} \sqrt{\mathrm{m}}$. It should be pointed out that the $K_{\mathrm{Ic} t h}$ estimation by the Griffith formula with the true surface energy $\gamma \cong \frac{\mathrm{Ea}}{20}$ as it was shown by Ashby [26] gives exactly the same results.

On the other hand, one can get an independent estimation of the value $K_{\text {Ic min }}=K_{\mu}$ for the real "coalescence" micromechanism of fracture with the help of formula (6) using realistic values of the parameters $X_{c}$ (e.g. equal to the average grain size [9]) and $\sigma_{f}^{*}$ (e.g. as a results of estimation using the specimens with different notches [22]): Hence, taking $X_{c}=30 \mu \mathrm{m}$ and $\sigma_{f}^{*}=2125 \mathrm{MPa}$ one can get $K_{\text {Ic } \min } \cong 20 \mathrm{MPa} V_{\mathrm{m}}$ observed in the experiments performed on steels and predicted by the $K_{\mu}$-model that corresponds to $\left(K_{\mathrm{Ic}} / E\right)_{\min } \geq 10^{-4} \sqrt{\mathrm{m}}$. 
The observed above difference in the estimates of the minimal possible fracture toughness which correspond to the available brittle fracture concepts, namely, the $K_{\text {Ic } \min }$ and $K_{\text {Ic } t h}$ values, is so large that it requires explanation. We shall try to give it using steels as an example. It is well known that grain boundaries in metals play a hardening part owing to two effects: barrier effect and the effect of multi-planar deformation. The first effect is stipulated by the very fact of the existence of cristallographic disorientation of neighbouring grains, and for purely geometrical reasons a dislocation cannot move from one grain to the adjacent one as a result of which the grain boundaries are said to be "unpenetrable" for dislocations. Something like this happens when a cleavage crack propagating in one grain approaches the boundary with another grain strongly disoriented with respect to the first one. Owing to the fact that the cleavage of crystals over their cleavage plane is the easiest way for their fracture and on the other side of the boundary the propagating crack cannot directly penetrate into such a plane, the crack is either arrested or requires additonal energy to be spent for fracture. It is evidented [27] that the main obstacle to the process of fracture of structural steels is the grain boundaries or other interfaces and it is suggested that the $\mathrm{K}_{\mathrm{Ia}}^{\mathrm{B}}$ value, i.e. fracture toughness for the crack arrest by the grain boundary, should be taken as a measure of their resistance to fracture. It is believed that the estimated difference between $K_{\mathrm{Ic} \min }$ and $K_{\mathrm{Ic} t h}$ values agrees with the above concept and the $K_{\mathfrak{I c} \text { th }}$ values should be considered as the minimal possible capability of the material to resist fracture during the cleavage crack propagation within the grain. The $K_{\mathrm{Ic} \text { min }}$ value characterizes the minimal fracture toughness of a polycrystalline material. Similar examples can be offered for other materials: cast irons with graphite of various shape, pipeline steels, ceramic materials with a complex phase composition [23].

Needless to say, many questions remain to be answered here, and primarily those concerning physical interpretation of parameters $X_{c}$ and $\sigma_{f}^{*}$ for materials with an intricate multiphase structure; how to modify the model for it to fit better the physical essence of the processes observed during fracturing of

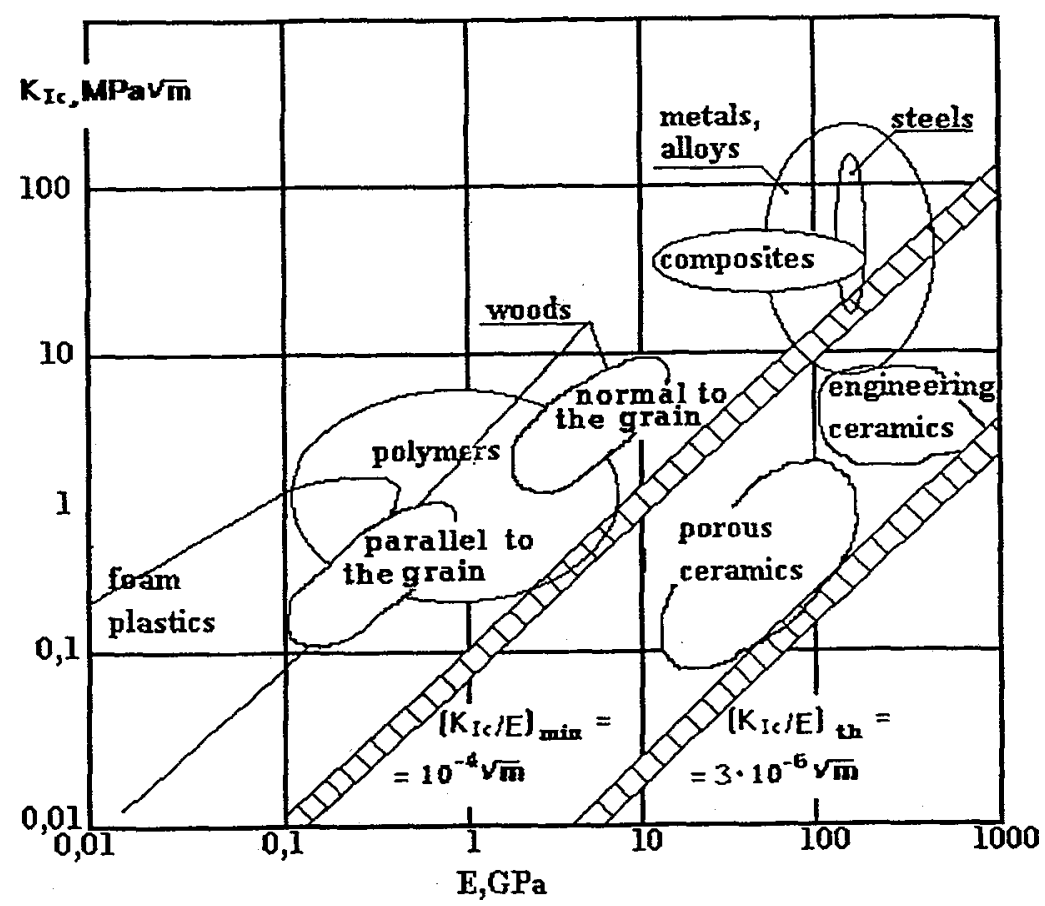

Figure 4: Estimation of the minimal $\left(K_{\mathrm{I} c} / \mathrm{E}\right)_{\min }$ and threshold $\left(K_{\mathrm{I} c} / \mathrm{E}\right)_{\text {th }}$ normalized fracture toughnesses using the $K_{\mu}$ -model against a background [26] of the arrays of experimental data for the main classes of engineering materials. 
different materials. Yet, the information presented above indicates that the $K_{\mu}$-model in the form proposed yields reasonable fracture toughness estimates and sets the investigator on the right track.

To confirm the above postulate consider a plot from Ashby's work [26] which illustrates the relation between fracture toughness and elasticity modulus for the main classes of engineering materials, Fig.4. The plot shows two inclined shaded bands: one of them corresponds to the above estimation of the threshold values $\left(K_{\mathrm{Ic}} / E\right)_{t h} \cong 3 \cdot 10^{-6} \sqrt{\mathrm{m}}$ predicted for the "decohesion" fracture (the band of a finite width is presented in order to take into account the properties of other materials); the other corresponds to $\left(K_{\mathrm{Ic}} / E\right)_{\text {min }} \geq 10^{-4} \sqrt{\mathrm{m}}$, predicted above for the "coalescence" fracture. As is seen from the plot, all experimental results for all materials lie above the line $\left(K_{\mathbf{I c}} / E\right)_{t h} \geq 3 \cdot 10^{-6} \sqrt{\mathrm{m}}$, i.e. this line represents the minimum fracture toughness for all materials. As to the line $\left(K_{\mathrm{Ic}} / E\right)_{\min } \geq 10^{-4} \sqrt{\mathrm{m}}$, it is evident that its position depends on the particular material structure triggering the "coalescence" fracture mechanism. As is seen, this line calculated for steels is in fair agreement with the experimental data array for steels approaching it very closely in the lower part, as could be expected. Estimation of the $\left(K_{\mathrm{Ic}} / E\right)_{\min }$ value for each group of materials in brittle state might be useful for a more detailed analysis of their fracture mechanisms and for the elaboration of recommendations for optimizing their structures and enhancing fracture toughness. First and foremost this concerns brittle materials.

\section{RELATION OF FRACTURE TOUGHNESS TO OTHER CHARACTERISTICS}

The fracture models are also useful in that they predict simple relationships between fracture toughness and other mechanical characteristics, such as yield stress, ultimate strength, uniform elongation, elongation and reduction of area at fracture, strain hardening exponent. Formula (4) which establishes the relation between fracture toughness and the yield stress can be an example of such relationship. As it has already been mentioned, with the proviso that a change in the yield stress is not caused by changes in the material structure, many experimental evidences were obtained of the validity of formula (4). In support of the above-stated, other works can be mentioned in which relation (4) in one or another particular case was substantially experimentally verified. Thus the experiments performed in [28] on a low-carbon steel and in [29] on a martensitic steel confirmed the relation

$$
K_{\text {Ic }}=A_{1} \sigma_{y}^{-1.5}
$$

which follows from Eq.(4) as a particular case for the material with hardening exponent $n=0.25$. In ref.[30] for shipbuilding steels the following relationship was obtained

$$
K_{\text {Ic }}=A_{2} \sigma_{y}^{-1.25}
$$

which follows from Eq.(4) at $n=-2 / 7$ and in ref.[31] for some other materials they got

$$
K_{\mathrm{Ic}}=\mathrm{C} \frac{\sigma_{\mathrm{fc}}^{3}}{\sigma_{\mathrm{y}}^{2}}
$$

which also corresponds to formula (4) at $n=0.2$.

The analysis of other fracture models which indicate the existence of fracture toughness relation with other mechanical properties can be found in refs. $[9,32]$. A generalized fracture model and a criterion which take into account the stress state at the crack tip are proposed in ref.[32]. The authors of that work obtained generalized relationships in a closed form which relate fracture toughness with other mechanical properties and they also show that as particular cases those relationships can yield results similar to those which follow from a number of previous models. In a number of practical cases the above relationships can be obtained for an approximate estimation of the expected fracture toughness of the material from its mechanical characteristic and for the express evaluation of the assumed fracture toughness of new materials being developed without performing expensive and tedious fracture toughness tests.

\section{CONCLUSIONS}

1. Of the two concepts of brittle fracture of the engineering materials based on the "decohesion" and "coalescence" fracture mechanisms, respectively, the first reflects the threshold fracture toughness for the materials of perfect structure, the second - the minimal fracture toughness of the material of a real structure. Since the "coalescence" fracture mechanism is observed with most engineering materials and requires higher fracture energy and the "decohesion" mechanism is a part of the "coalescence" mechanism, 
it is necessary to investigate both of them in order to study the nature of the fracture process and to optimize the material structure.

2. The model parameters on which the "coalescence" fracture mechanism is based, namely, characteristic distance $X_{c}$ and microcleavage stress $\sigma_{f}^{*}$ are directly related to the material minimal fracture toughness and are defined by the weakest elements of its microstructure.

3. Rigorous physical interpretation of the characteristic distance and microcleavage stress requires statistical (dimensional and orientation) consideration, yet modelling of the fracture process in mean values of the above parameters seems to be useful.

4. Fracture toughness dependences on the temperature and loading rate both for a number of ceramic materials and for steels in the brittle-to-ductile transition region have much in common. For this reason it is possible to use some fracture models, and in particular, $K_{\mu}$ - model to analyze fracture of ceramic materials and to optimize their structure.

5. The main ways of enhancing fracture toughness of engineering materials are associated not only with the plasticization of the latter but also with the creation of such structures which would contribute to the increase of their minimal fracture toughness values. And this can be achieved by increasing each of the two fundamental parameters of the material fracture micromechanism: characteristic distance and cleavage stress.

\section{ACKNOWLEDGEMENTS}

Support to this research by the European Community COPERNICUS programe, contract CIPA CT94 0194 is gratefully acknowledged.

\section{REFERENCES}

[1] Griffith A. Phil.Trans. Roy. Soc. London A 221 (1920) 163-198.

[2] Barenblatt G.I. I: $P M M 23$ (1959) 3, 242-249. II: PMM 23 (1959) 4, 631-637. III: PMM 23 (1959) 5, 940-946 (in Russian).

[3] Leonov M.Ya., Panasyuk V.V. Prykl. Mekhanika 4 (1959) 391-401 (in Ukrainian).

[4] Pisarenko G.S., Krasowsky A.J., Vainshtok V.A. Engineering Fracture Mechanics 28 (1987) N $5 / 6,539-554$.

[5] Orowan E. Repts. Progr. Phys. 12 ( 1948) N11, 185-199.

[6] Neuber H. Konstruction 20 (1968) 245-251.

[7] Tetelman A.S., Wilshaw T.R. and Rau C.E. Internat. Journ. Fracture Mechanics 4 (1968)147-157.

[8] Pisarenko G.S. and Krasowsky A.J. "Analysis of kinetics of quasibrittle fracture of crystalline materials". Mechanical Behavior of Materials. Proc. Int. Conf. ICM-1, Kyoto 1971(Kyoto, JSMS,1972) vol.1, pp. 421-432.

[9] Krasowsky A.J. Brittleness of Metals at Low Temperatures (Naukova Dumka Ed., Kiev, 1980) 340 (in Russian).

[10] Cherepanov G.P. - Prikladnaya mathematika i mechanika 31 (1967) 3, 432-436 (in Russian).

[11] Cherepanov G.P. Mechanics of brittle fracture (Moscow: Nauka, 1974) 640 (in Russian).

[12] Rice J.R. and Rosengren G.F. J. Mech. \& Phys. Solids 16 (1968) N1, 1-12.

[13] Ritchie R.O., Knott J.F. and Rice J.R J. Mech. \&. Phys. Solids 21(1973) N6, 395-410.

[14] Curry D.A. (Dusseldorf, VDI, Fortschr.-Ber., 1979 ) VDI-Z, Reihe 18, N6, 214-225 (cited from [21]).

[15] Beremin F.M. Metall. Transactions 14A (1983)2277-2287.

[16] Wallin K. In Defect Assessment in Components- Fundamentals and Applications, ESIS/ECF9?Eds. J.D. Blauel and K-H. Schwalbe (London,Mech. Engng. Publ.1991) 415-445.

[17] Pluvinage G., Krasowsky A.J., Krassiko V.N. Memoires et Etudes Scientifiques Revue de Metallurgie, Mars (1992) 137-152.

[18] Pisarenko G.S. and Krasowsky A.J. "Analysis of criteria for ultimate state of material at the crack tip in brittle fracture of metals". Proc. Second Internat.Conf. Mechanical Behavior of Materials, 16-20 Aug. 1976, (ASM, Boston, 1976) Spec. vol., pp.348-376 .

[19] Gerberich W.W., Chen S.-H., Lee C.-S. Metall. Transactions 18A (1987) 1861- 1875.

[20] Meizozo A.M., Ibabe I.M.R., Sole V. and Gil-Seviliano J. "Brittle fracture nucleation in SA533 B-1 steel". In: Fracture control of Engineering Structures, Proc. ECF-6, Amsterdam, June 15-20, 1986 (EMAS-Publ., Amsterdam, 1986) pp.1763-1773. 
[21] Kotilainen H. "The micromechanisms of cleavage fracture and their relationship to fracture toughness in a bainitic low-alloy steel" Thesis Dr. Techn. (Helsinki University of Technology, Otaniemi, Techn.Res.Centre of Finland, Publ.23, ESPOO 1980) 133 p.

[22] Dahl W., Dormagen D. and Halim A. "Micromechanisms of fracture". In: Fracture Control of Engng.Structures. Proc.ECF-6, Amsterdam, June 15-20, 1986 (EMAS-Publ., 1986) pp.1467-1489.

[23] Krasowsky A.J. "Local approach to the fracture of engineering materials". In Fracture: A Topical Encyclopedia of Current Knowledge Dedicated to Alan Arnold Griffith, Ed. G. Cherepanov (Krieger Publishing Company, Melbourne, USA, 1996) pp. 341-358.

[24] Pineau A. "Global and local approaches of fracture - transferability of laboratory test results to components" Topics in Fracture and Fatique. Ed. A.S. Argon.( Springer-Verlag, 1992) pp. 197-234.

[25] Pisarenko G.S., Krasowsky A.J. and Yokobory T. Reports of Res. Inst. Strength and Fracture of Mater. Tohoku University, Sendai, Japan 13 (1977) N1, 1-57.

[26] Ashby M.F. Acta metall. 37 (1989) N5, 1273-1293.

[27] Hahn G.T.. Met. Transactions 15A (1984) N1, 947-959.

[28] Krafft J. and Sullivan A.M.. Trans. ASM 56 (1963) N1, 160-175.

[29] Silva da P.S.P. and Brook R. "Fracture toughness of martensitic steels" Mechanical Behavior of Materials, Proc. Int. Conf. ICM-1. Kyoto, 1971 (Kyoto, JSMS,1972) vol.1, pp.513-524.

[31] Pandey R.K., Haridas J.D. and Banerjee S. Engng. Fract.Mech. 6 (1974) N1, 105-118.

[32] Krasowsky A.J. and Vainshtok V.A. Problemy Prochnosti (1978) N5, 64-69 (in Russian). 